\title{
A Study on Detection Method of Falling Symptoms
}

\author{
Jin Zhang, Takuya Tajima \\ Fukuoka Institute of Technology Graduate School, Fukuoka Institute of Technology, Fukuoka, Japan \\ Email: mjm19201@bene.fit.ac.jp
}

How to cite this paper: Zhang, J. and Tajima, T. (2020) A Study on Detection Method of Falling Symptoms. Journal of Computer and Communications, 8, 73-82. https://doi.org/10.4236/jcc.2020.812007

Received: August 3, 2020

Accepted: December 17, 2020

Published: December 24, 2020

\begin{abstract}
The purpose of this study is to prevent the occurrence of falls by defining the symptoms of falls and detecting them in advance. The focus of the study is on the specific definition of "Falling Symptoms". In this regard, we conducted a questionnaire survey on people of different ages to obtain the state of themselves and their surroundings when they fell down. In addition, we used the elderly simulated experience kit to achieve the purpose of using young people to replace the elderly. Young people were asked to walk on different roads in different shoes with and without elderly simulated experience kit and photographed them with a high-speed camera to observe the changes of their muscles and joints. We also simulated the movement of center of gravity of the people with and without elderly simulated experience kit by a pressure distribution sensor mat.
\end{abstract}

\section{Keywords}

Falling Symptoms, Questionnaire, High-Speed Camera, Center of Gravity, Elderly Simulated Experience Kit

\section{Introduction}

According to the White Paper on Aging Society by the Cabinet Office of the first year of Reiwa, the total population of Japan as of October 1, 2018 was 126.44 million [1]. The population over the age of 65 was 35.58 million, and the ratio (aging rate) to the total population was $28.1 \%$. Of the population aged 65 and over, the "65 - 74 years old" is 17.6 million (8.4 million males and 9.2 million females), accounting for $13.9 \%$ of the total population, and the "75-year-old population" is 1798 . The number of people ( 70.60 million men, 10.92 million women) was $14.2 \%$ of the total population, surpassing the population aged 65 to 74 for the first time. In the future, it is estimated that in 2065, about 2.6 people 
will be over 65 years old, and about 1 out of 4 people will be over 75 years old, and the proportion of the population over 75 years old will be $25.5 \%$. The ratio is that 1.3 active generations support one person over the age of 65 . Under such circumstances, the number of elderly people who need nursing care is increasing.

$80 \%$ of the causes of urgent transportation of elderly people are falling [2], and it can be said that there is a high possibility that stumbling and falls that may occur in everyday life will lead to serious injuries [3].

Therefore, by obtaining the information of the person's environment and the changes in various aspects of the body at the time of the fall [4], analyzing the internal and external causes of the fall [5], and thus defining the precursors of a fall in specific terms, is of great significance for preventing the occurrence of falls.

\section{Experiments and Questionnaires}

\subsection{Questionnaires}

A questionnaire survey was conducted to investigate the condition with the highest frequency of falls as an external factor of falls. The subjects of the questionnaire are students of Fukuoka Institute of Technology and residents around the university. Data were collected and analyzed about the age, sex, existence of falling experience in the past year, existence of the injury, fall place, fall time and fall cause. The content of the questionnaire includes age, gender, experience of falls within the past year, presence of injury, location of fall, time zone of fall, cause of fall, etc.

Since many people have experienced falls, including stumbling, it was found that stumbling occurred at any age [6], and a questionnaire survey was conducted to obtain detailed data specific to stumbling. The questionnaire used a paper-based survey and a Google form survey together.

\subsection{Elderly Simulated Experience Kit}

Experiments have shown that the physical functions of young people wearing elderly Simulated Experience Kit are similar to those of the elderly [7].

We used SANWA Elderly person simulated experience teaching material expert set III to simulate the state of the elderly (Figure 1). Supporters are attached to the knees and elbows to limit the range of motion, the weight band at the wrists and ankles makes the body heavy, and the vest with a weight forcefully puts the user in a leaning posture. It is a tool used to experience the posture and movement of a person.

Figure 2 shows the maximum bending angle when the kit is installed.

The knee and elbow supporters limit the range of motion, making it difficult to bend the knee and elbow at right angles (Figure 3).

\subsection{High-Speed Camera}

As internal factors of a fall, for what kind of posture when the stumbled to 


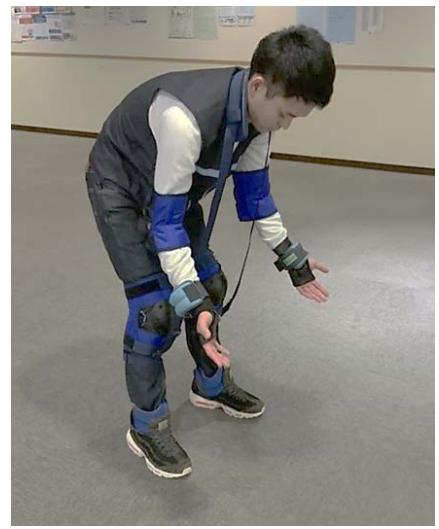

Figure 1. A state of wearing the elderly simulated experience kit.

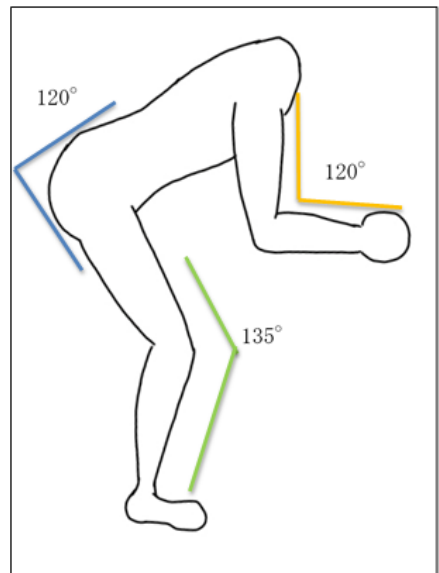

Figure 2. Maximum bending angle of each joint when the kit is installed.
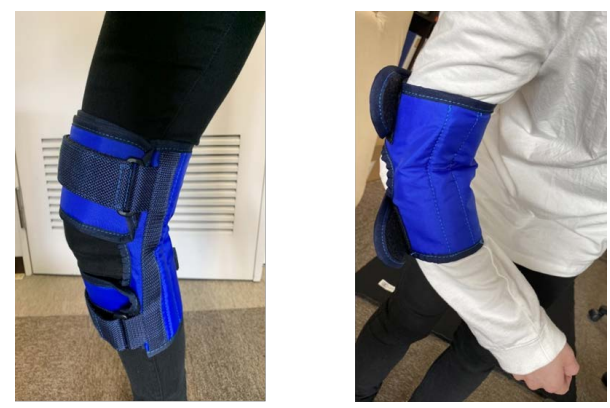

Figure 3. Maximum range of motion of the knee and elbow when the kit is installed.

analyze whether the lead to the fall, to shoot the moment of falling or stumbling with a high-speed camera. The degree of joint bending and the appropriate toe height are calculated based on the captured images. Similarly, we give examples of how to walk easily and features, compare them, and propose a better way of walking.

The model of the high-speed camera is JVC HD MEMORY CAMERA GC-LJ25 (Figure 4) [8]. The shutter speed is auto, the recording setting is 300 fps, and $640 \times 360$ pixels. Connect the camera to the iPad by direct connection and operate it using the special application "Sports Coaching CAM" [9]. 


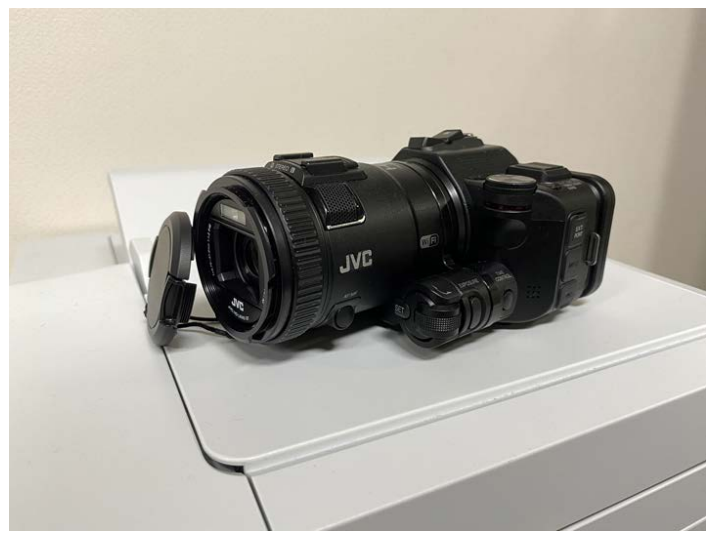

Figure 4. JVC HD MEMORY CAMERA GC-LJ25.

\subsection{Pressure Distribution Sensor Mat}

As internal factors fall, what walking in order to analyze whether the likely fall to investigate the movement of the center of gravity during walking using the pressure distribution sensor mat.

The equipment used in the experiment is pressure distribution measuring device FSA/BodiTrak bed system (Figure 5). The sensors are arranged vertically and horizontally 1 inch square, are thin and have excellent flexibility, and even small objects can detect contact within the range that can be read by one sensor (Table 1).

Pressure distribution sensor mat control software is FSA Software Ver 4.1.001. The electric signal of the pressure distribution sensor mat is displayed on the map as pressure data. At the same time, the line connecting the pressure center points in time series (pressure center line) and the pressure data in 3D map are displayed. It is also possible to change the measurement speed and edit the cut and paste.

A pressure distribution sensor mat (BodiTrak) is laid on a flat floor, a distance of $30 \mathrm{~cm}$ is taken from the edge of the mat, and walking is started from that point (Figure 6). The subjects were four men and four women, all around the age of twenty. When walking, ask them to take off their shoes and put them on socks. In addition, in the case of the elderly simulation experience kit is not mounted, and the case that reproduces the walking for the elderly at the time of mounting is measured and compared. Do not give detailed instructions to the subject and simply say, "Please walk normally".

\section{Experiment and Questionnaire Results}

\subsection{Questionnaire Results}

Among men and women in their $20 \mathrm{~s}, 20 \%$ have experienced a fall, and among elderly people aged 60 to 80 , as many as $46 \%$ have experienced a fall. In addition, among the elderly who have experienced falls, the proportion of injuries such as broken teeth or facial injuries has reached $66 \%$. Therefore, it can be seen that the elderly are more likely to fall and get injured. 


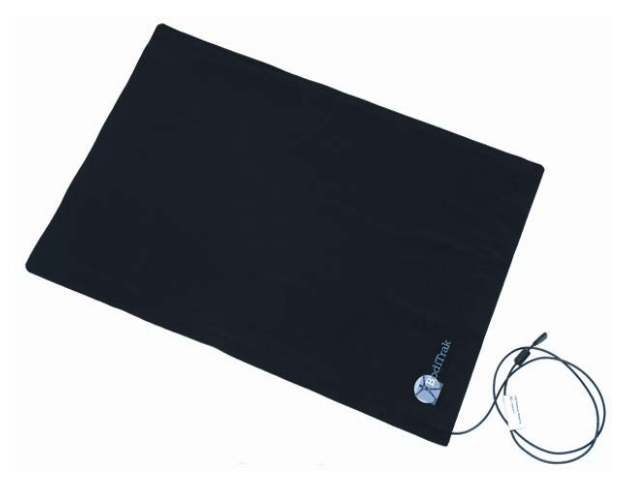

Figure 5. FSA/BodiTrak.

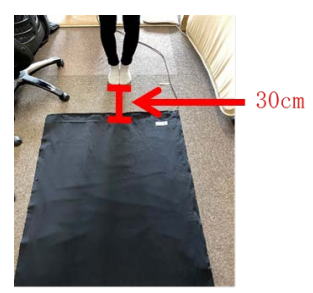

Figure 6. Start position of walking.

Table 1. Sensor specifications [10].

\begin{tabular}{cc}
\hline Matte size $(\mathrm{mm})$ & $2032 \times 863$ \\
Number of sensors & $64 \times 27=1728$ \\
Sensor size $(\mathrm{mm})$ & $25.4 \times 25.4$ \\
Thickness $(\mathrm{mm})$ & 2.5 \\
\hline
\end{tabular}

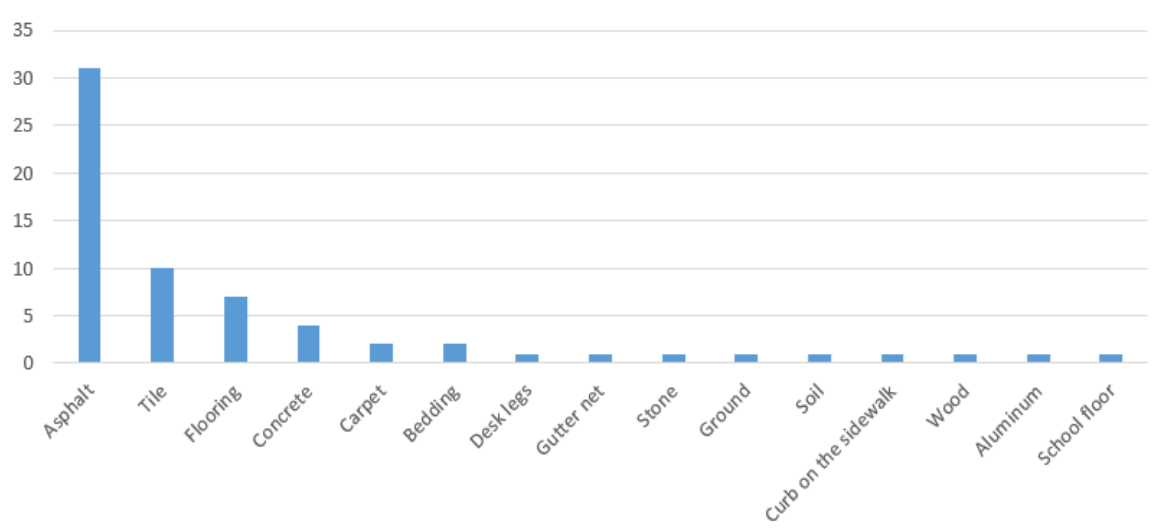

Figure 7. The material of the floor surface when stumbled.

The material of the stumbled area is asphalt, tiles, flooring in order (Figure 7).

The most worn ones were sneakers, sandals and slippers were also mentioned (Figure 8).

The causes of stumbling without being caught by things were the most inattentiveness such as being dim, looking away, and looking at the smartphone, followed by physical problems such as the leg not rising (Figure 9). 


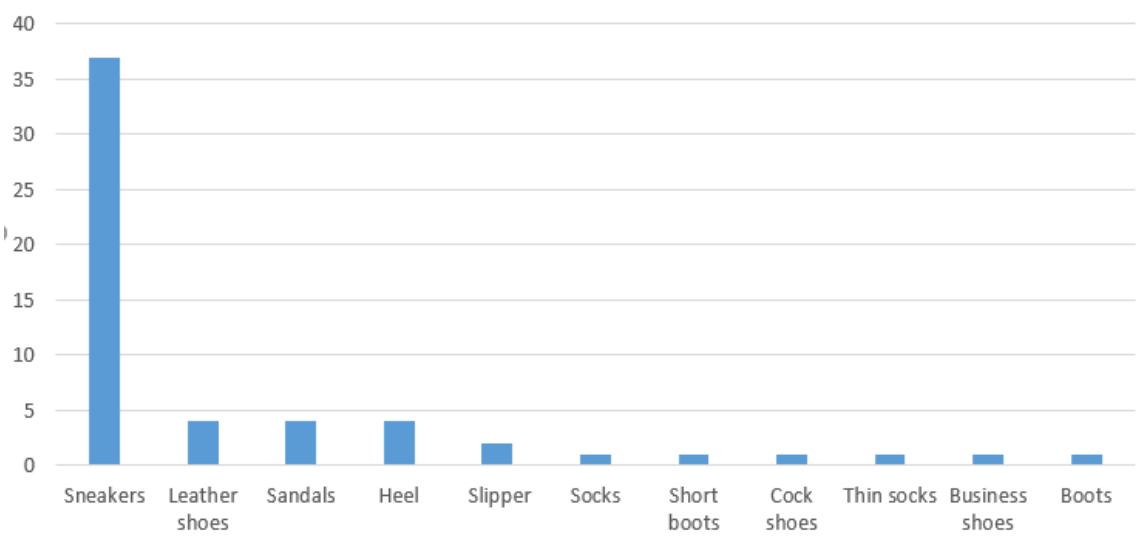

Figure 8. The type of shoes worn when the fall occurred.

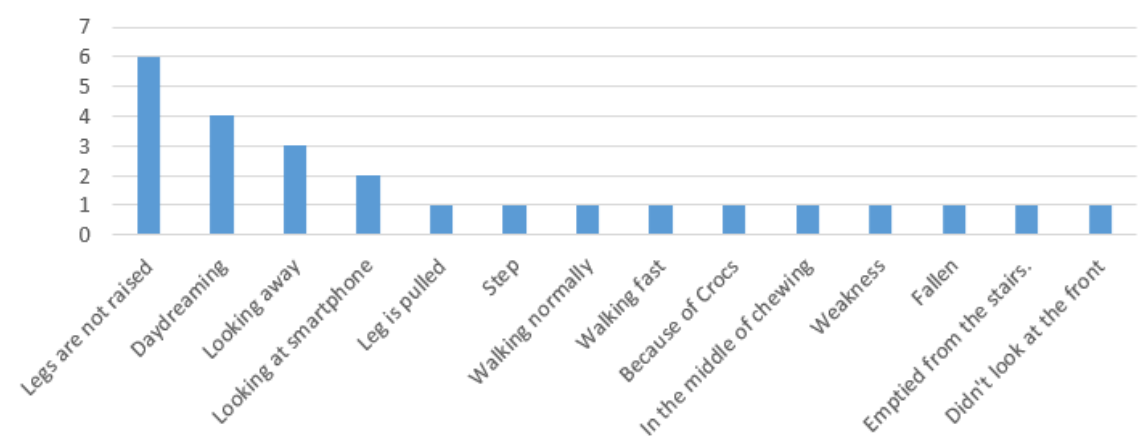

Figure 9. The causes of stumbling.

\subsection{Analysis of the Captured Video}

Instantly fall or stumble on a high-speed camera to analyze, when tripped flat ground without obstacles occur, cause of stumbling roughly divided into two types. The first is rubbing the toes against the ground, and the second is rubbing the soles of the feet against the ground.

When the toes rub the ground, it was found that a stumbling occurred when the heel of the stepped foot was raised too much (Figure 10).

When the sole of the foot rubs the ground, it was found that the sole of the foot was parallel to the ground for a long time, the knee was not raised, and just before stumbling, the patient leaned forward more than usual (Figure 11).
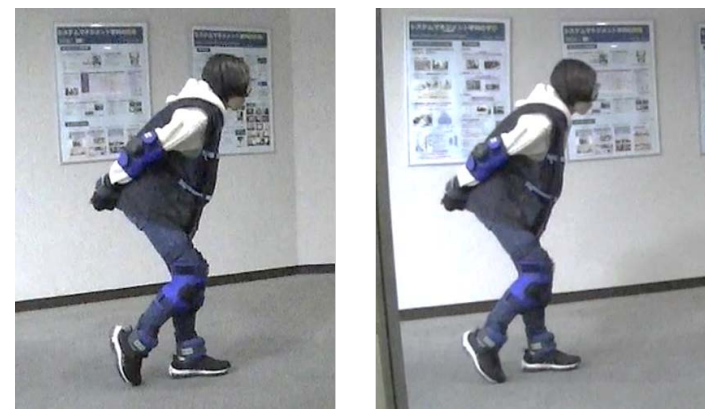

Figure 10. The moment of tripping caused by toes (left) and walking normally (right). 


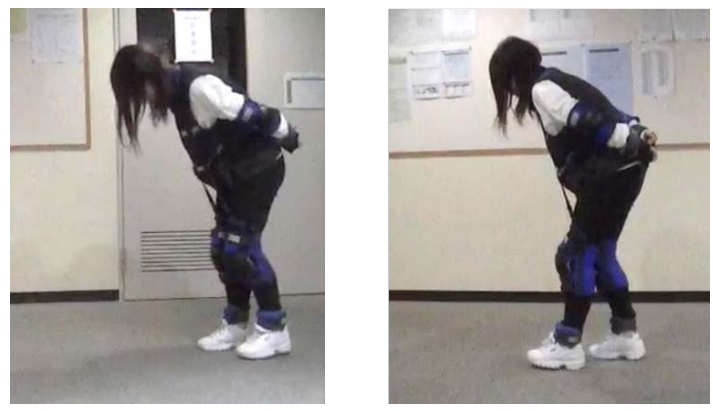

Figure 11. The moment of tripping caused by sole of the foot (left) and walking normally (right).

\subsection{Analysis of the Movement of the Center of Gravity}

The narrower the movement range of the center of gravity coordinate and the smaller the support base [11], the easier it is to lose balance. The base surface of the support is an area where a line connects the parts where the body and the support are in contact with the floor surface (Figure 12). The stability limit is the limit region where the center of gravity of the body can be separated from the center of the supporting base without losing the balance.

Therefore, we focus on the movement of the barycentric coordinates and consider the balance during walking. Figures 13-15 show the barycentric coordinates detected by the pressure distribution sensor mat in chronological order from bottom to top (hereinafter referred to as the pressure center line), each of which is a 20-year-old subject's kit. It is classified by the presence/absence of wearing and the gender without wearing the elderly simulated experience kit.

Generally, the greater the variation of the pressure center line, the wider the stability limit and the more difficult it is to fall. Wearing the simulated elderly experience kit increased the number of steps and widened the support base (Figure 13).

In addition, in comparison with gender, the pressure center line is slightly linear for women overall (Figure 14).

However, the pressure center line of some men is similar to that of women in the number of steps, stride length, and movement of the center of gravity (Figure 15).

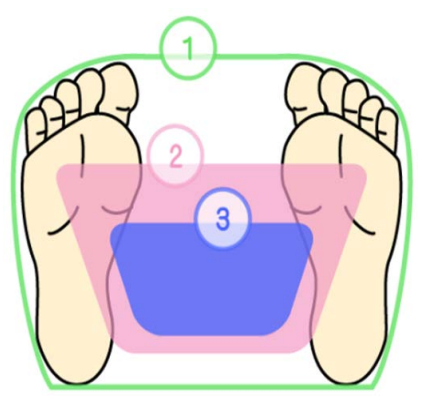

Figure 12. Support base and stability limit (1): Support base; (2): Sability limit of youth; (3): Sability limit of elderly). 

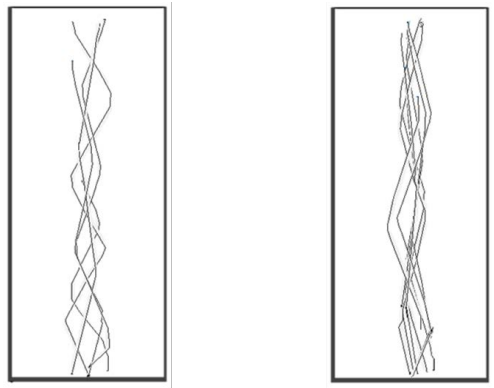

Figure 13. The pressure center line when the subject is wearing and not wearing kit.
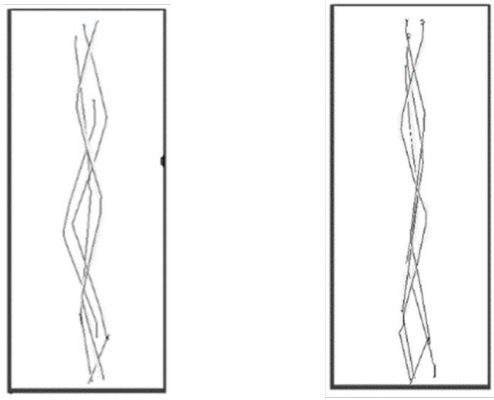

Figure 14. The pressure center line of men (left) and women (right).
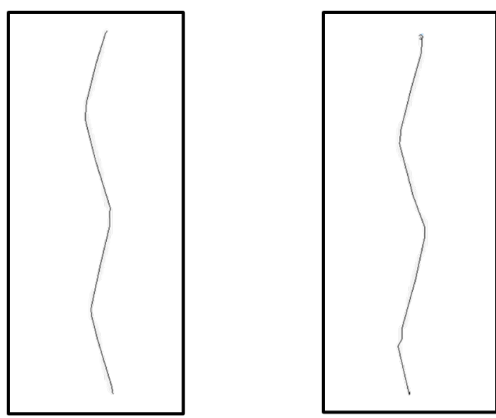

Figure 15. Similar pressure center lines.

\section{Conclusions}

From the results of a questionnaire about the experience of falls including stumbling, it was found that there were few falls in the twenties, but stumbling, which is one of the signs of a fall, occurred regardless of age, only by preventing the fall by muscle strength. It is thought that young people are always distracted because the causes of stumbling in their $20 \mathrm{~s}$ are many carelessnesses such as watching smartphones and the time zones are different. In addition, we think that the reason why respondents in their thirties or older stumble in the evening is that they are less likely to use their muscles due to desk work, etc., so that their muscles become rigid and their legs do not rise easily. Since leaning forwards regardless of age affects stumbling, we think that training the erector spinae muscles and latissimus dorsi that enforces forward leaning may be one of the measures to prevent falls. Stumbling often occurs when wearing sneakers in all ages, because many of the respondents are young, and even older people are choosing 
sneakers that are easy to use [12]. Conceivable. In other words, since there are many opportunities to wear sneakers regardless of age, we think that sneakers have become more frequent.

It can be inferred that in the case of the elderly, the muscle strength declines and the person cannot stumble on a stumbling block [13], leading to a fall. Most of the youthful sickness was found to be leaning forward due to the habit of looking at a smartphone and the floating fingers caused by the stoop, which reduced the ability to kick with the toes.

It can be said that the elderly are more likely to fall because the range of motion of their joints is narrow and the load of weight associated with muscle weakness is applied [14]. From the results of the pressure distribution sensor, when young people use the elderly simulated experience kit to reproduce the walking of the elderly, the range of motion and muscular strength is limited, but a balance is established to withstand that posture, and a stability limit is set. It is thought to be supplemented. Moreover, it is generally said that the women with introcrum have a linear pressure center line and are more likely to trip over. However, when comparing the pressure center lines among some male and female subjects, no significant difference was observed. Therefore, it is difficult to identify or classify sex from the data obtained this time. In addition, females are not always groin, and males do not necessarily stumble because the stability limits are wide. In other words, it is difficult to propose preventive measures for each sex because there is no difference in the frequency of trips by gender.

Analysis with the high-speed camera revealed that it is important to keep the angle of the ankle as sharp as possible without leaving the pulling foot behind the body. By keeping the ankle at an acute angle, it is possible to reduce the time for the toes to rub the ground and the time for the sole to be parallel to the ground. However, no matter how much you keep the ankles of your feet sharp, you cannot avoid stumbling on your soles unless your knees are raised. Therefore, it is necessary to raise the stepped foot to a high level and keep the ankle at an acute angle while keeping the foot that pulls in behind as much as possible.

\section{Conflicts of Interest}

The authors declare no conflicts of interest regarding the publication of this paper.

\section{References}

[1] Cabinet Office (2020) Reiwa 1st Year White Paper, Chapter 1, Section 1, Status of Aging. (Japanese) https://www8.cao.go.jp/kourei/whitepaper/w-2019/zenbun/pdf/1s1s_01.pdf

[2] Cabinet Office (2020) Reiwa 1st Year White Paper, Chapter 1, Section 2, Trends in Living in the Elderly, 28-32. (Japanese) https://www8.cao.go.jp/kourei/whitepaper/w-2019/zenbun/pdf/1s2s_02_01.pdf

[3] Tokyo Fire Department (2020) <Safety and Security> <Topics $>$ Accidents of the Elderly Seen from Emergency Transport Data. (Japanese) 
[4] Kong, X.B., Meng, Z.L., Meng, L. and Hiroyuki, T. (2019) Three-States-Transition Method for Fall Detection Algorithm Using Depth Image. Journal of Robotics and Mechatronics, 31, 88-94. https://doi.org/10.20965/jrm.2019.p0088 https://ci.nii.ac.jp/naid/130007602033/10.20965/jrm.2019.p0088

[5] Kyaw, T.G., Choksuriwong, A. and Suvonvorn, N. (2018) Soft Fall Detection Using Multi-Sensors. Proceedings of Asia Pacific Conference on Robot IoT System Development and Platform, 25 December 2018, 52-53.

https://ci.nii.ac.jp/naid/170000178807/

[6] Japan Fall Prevention Society. (Japanese) http://www.tentouyobou.jp/senmonka/teigi.html

[7] Hirano, M., Miyaji, Y., Takeda, K. and Nakagawa, K. (2019) The Effect of Wearing a Simulated Experience Set on Life Functions and Posture Control of College Students. Journal of Health and Medical Science, 9, 83-93. (Japanese) https://ci.nii.ac.jp/naid/120006648820/

[8] Video Camera GC-LJ25B User Guide. (Japanese) http://manual3.jvckenwood.com/c3z2/lj25jp/

[9] JVC GC-LJ25B. (Japanese) https://www3.jvckenwood.com/pro/video/gc-lj25b/spec.html

[10] Pressure Distribution Measuring Device FSA/BodiTrak. (Japanese)

[11] Stability Limit/Center of Foot Pressure. (Japanese) https://physioapproach.com/blog-entry-492.html

[12] Ministry of Health, Labor and Welfare (2020) Safety Shoes Effective for Fall Prevention. (Japanese) https://www.simon.co.jp/fall_disaster_shoes

[13] Asakawa, K. (2003) Elderly Strength and Strength Training. Physical Therapy Science, 18, 35-40. (Japanese)

[14] Cai, W.-Y., Guo, J.-H., Zhang, M.-Y., et al. (2020) GBDT-Based Fall Detection with Comprehensive Data from Posture Sensor and Human Skeleton Extraction. https://doi.org/10.1155/2020/8887340 